\title{
Transformation maligne sur cheilite actinique chronique chez un sujet roux
}

\author{
Tommaso Lombardi ${ }^{1}$, Jacky Samson ${ }^{2, *}$ \\ Laboratoire d'Histpathologie buccale, École de Médecine dentaire, Genève, Suisse \\ Division de Stomatologie et Chirurgie orale, École de Médecine dentaire, Genève, Suisse \\ jacky.samson@unige.ch
}

(Reçu le 6 juin 2013, accepté le 20 août 2013)

Mots clés :

cheilite actinique / OIN / rayons ultraviolets / facteurs favorisants

Key words:

actinic cheilitis / OIN / UV radiations / favoring factors
Résumé - La cheilite actinique chronique se traduit par un aspect clinique assez caractéristique. Elle survient après une exposition prolongée aux ultraviolets et aux intempéries et touche principalement la demi-muqueuse labiale inférieure. Elle constitue la principale cause d'OIN (Oral Intraepithelial Neoplasia) de la lévre et le risque de transformation maligne dépend de la dose de rayons ultraviolets et de facteurs favorisants individuels. Toute lésion faisant suspecter un foyer de transformation doit être traitée de préférence par excision.

\begin{abstract}
Malignant transformation of chronic actinic cheilitis occurring in a redhead man. Chronic actinic cheilitis usually presents with a rather characteristic clinical aspect. This condition occurs after long-term exposure to sunlight and other chronic irritating factors (wind, humidity, and smoking). Lesions mainly affect the lower vermilion and represent the more important aetiology of OIN (Oral Intraepithelial Neoplasia) of the lip. The risk of malignant transformation is related to the amount of absorbed ultraviolet radiation and linked to personal favouring factors. A lesion suspicious to have undergone to malignant transformation should be preferentially totally excised.
\end{abstract}

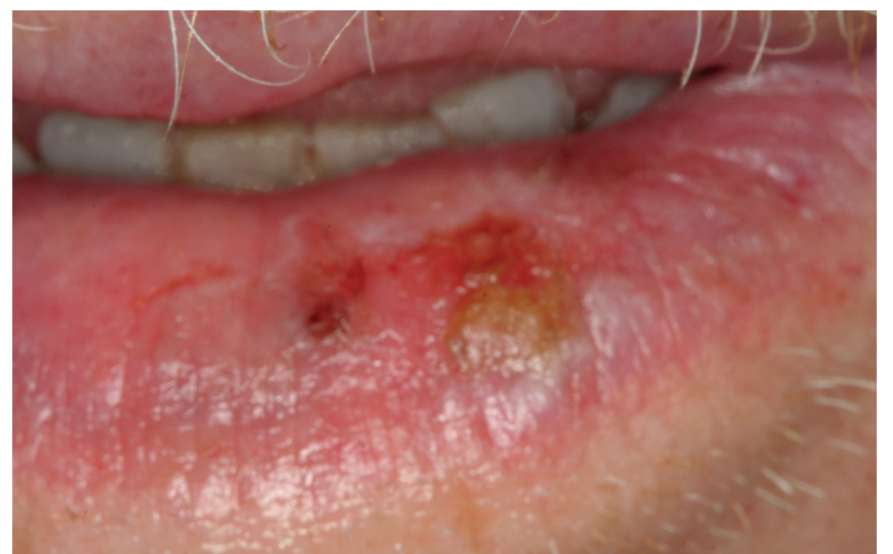

Fig. 1. Croûte sur la demi-muqueuse labiale inférieure présente depuis 6 mois. Noter l'aspect remanié de la demi-muqueuse dans son ensemble faisant évoquer une cheilite actinique.

Fig. 1. Scab localised on the lower vermillion of 6 months duration. Notice the remodelling of all mucosa of the vermilion evocating a chronic actinic cheilitis.

Un patient, âgé de 70 ans, professeur de musique à la retraite, qui a très peu fumé, est venu consulter pour une croûte sur la demi-muqueuse labiale inférieure, douloureuse, persistant depuis 6 mois, siégeant à $1,5 \mathrm{~cm}$ de la commissure gauche. À l'interrogatoire, on ne retrouvait pas d'antécédents personnels ou familiaux. C'était un sujet roux, aux yeux bleus, avec des taches de rousseur, qui s'était peu exposé au soleil en raison de la sensiblité de sa peau. Il présentait un remaniement de l'ensemble de sa demimuqueuse labiale inférieure (disparition de la limite cutanéo-muqueuse, présence de plages kératosiques associées à des plages atrophiques, érythémateuses ou érosives) faisant néanmoins suspecter une cheilite actinique. Il existait également une large zone située, près de la commissure gauche, où le remaniement semblait plus prononcé. Cette zone dont la limite postérieure, assez régulière, était soulignée par une bande kératosique, comportait 2 plages érosives et une lésion croûteuse (Fig. 1). Il n'y avait aucune zone indurée à la palpation.

Cet aspect faisant évoquer un foyer de transformation maligne, toute la zone suspecte a été excisée ; la pièce d'exérèse mesurait 1,5 $\times 0,8 \times 0,3 \mathrm{~cm}$. L'examen histopathologique de cette pièce a confirmé qu'il s'agissait bien d'une cheilite actinique. On observait un épithélium malpighien irrégulier, tantôt aminci, 


\section{A S C L I N I Q U E}

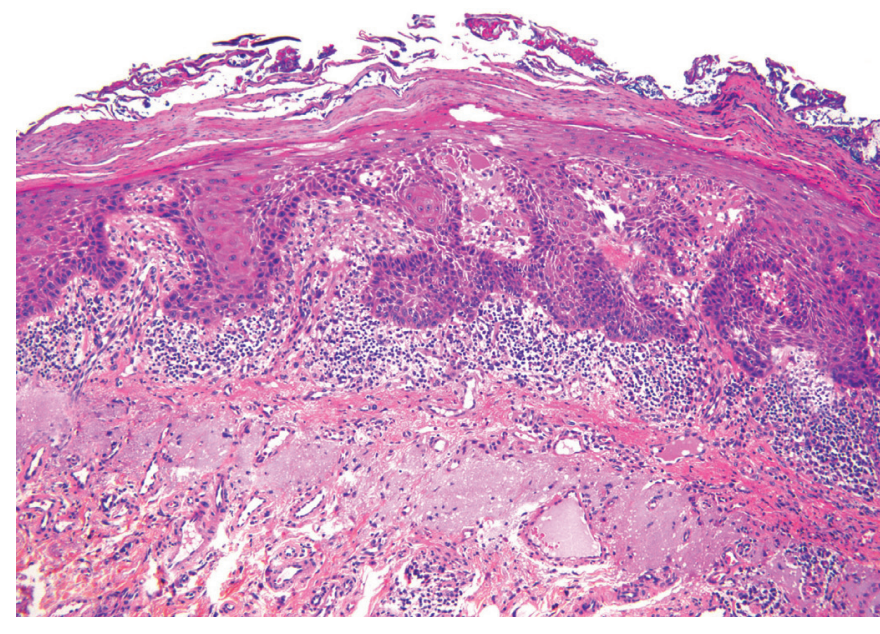

Fig. 2. Hyperkératose et hyperplasie épithéliale irrégulière. Les altérations cytologiques intéressent plus des deux tiers de la hauteur épithéliale totale (0IN3). Le chorion est le siège d'un dense infiltrat inflammatoire lymphoplasmocytaire et d'une élastose actinique (HE $\times 10)$.

Fig. 2. Hyperkeratosis and irregular epithelial hyperplasia. Atypical cells occupy more than the two-thirds of the epithelium (OIN3). The corium contains a dense lymphoplasmocytic infiltrate and shows amorphous, acellular, basophilic changes $(H E \times 10)$.

tantôt hyperplasique, comportant par endroits en surface une épaisse couche parakératosique. Le chorion était le siège d'une élastose actinique et d'un infiltrat inflammatoire lymphoplasmocytaire, parfois dense (Fig. 2). Le centre de la pièce était occupé par une ulcération avec des altérations dysplasiques de haut grade (OIN3). La plupart des limites de l'excision passait en zone d'OIN de bas grade (OIN1).

Le patient a été informé de la nature de la lésion et du risque potentiel de transformation maligne de sa cheilite actinique. Comme il venait de quitter Genève pour emménager en moyenne montagne, il lui a été recommandé d'être très vigilant et d'appliquer une protection anti-solaire efficace dès que nécessaire, et d'effectuer régulièrement tous les 6 mois un examen de contrôle.

La cheilite actinique chronique se traduit par un aspect clinique de la demi-muqueuse assez caractéristique (sécheresse, desquamation, modification de la couleur, plages kératosiques, jaunâtres, érythémateuses, atrophiques ou érosives, parfois des fissures chroniques, et toujours une limite cutanéo-muqueuse irrégulière) mais c'est l'examen histopathologique qui confirme le diagnostic en révèlant les remanienents caractéristiques : atrophie de l'épithélium avec hyperkératose plus ou moins importante, élastose actinique avec homogénéisation des fibres collagènes du chorion qui deviennent basophiles, présence de télangiectasies et d'un très discret infiltrat inflammatoire chronique. Elle touche presque toujours la lévre inférieure et s'observe surtout chez des sujets qui sont exposés professionnellement au soleil et aux intempéries; l'effet est plus marqué en montagne ou sur la mer [2]. La cheilite actinique constitue la principale cause d'0IN (oral intraepithelial neoplasia) de la lévre inférieure [5] et le risque de transformation en cancer varie de $1 \%$ à $20 \%$ [4]. Le foyer de transformation maligne se révéle fréquemment par une lésion croûteuse persistante [4] comme dans le cas rapporté. Le risque de transformation maligne dépend principalement de la dose de rayons ultraviolets mais il existe des facteurs favorisants liés à des anomalies de la peau qui prédisposent aux cancers cutanés : ce sont le xeroderma pigmentosum [1], l'albinisme [6] et les sujets roux [3] ; pour ces sujets, l'atteinte labiale survient presque toujours après de nombreux cancers cutanés.

Devant un foyer de transformation maligne sur cheilite actinique chronique, de nombreux traitements ont été proposés : 5-fluorouracil, imiquimod, diclofénac dans un gel d'acide hyaluronique, exérèse chirurgicale, électrocoagulation, cryochirurgie, laser, thérapie photodynamique [6]. Seule l'exérèse chirurgicale permet de contrôler la qualité du traiement. Pour l'exérèse chirurgicale, de nombreux auteurs proposent une vermillonectomie qui semble rarement indiquée car la transformation maligne est le plus souvent limitée à une zone étroite et une exérèse préventive de la cheilite actinique est rarement justifiée. Après le traitement, le patient doit être contrôlé régulièrement.

Le traitement comporte aussi des mesures préventives qui doivent être rappelées de façon insistante à tous les sujets exposés aux ultraviolets, et impérative à ceux qui présentent des facteurs prédisposants : limiter l'exposition solaire et utilisation régulière d'écran solaire avec un indice élevé de protection.

\section{Conflits d'intérêt : aucun}

\section{Références}

1. Beogo R, Andonaba JB, Bouletreau P, Traore Sawadogo H, Traore A. Xeroderma pigmentosum révélé par des carcinomes épidermoïdes multiples de la face chez un enfant. Rev Stomatol Chir Maxillofac 2012;113:50-2.

2. de Souza Lucena EE, Costa DCB, da Silveira EJD, Lima KC. Prevalence and factors associated to actinic cheilitis in beach workers. Oral Dis 2012;18:575-9.

3. Ferrucci LM, Cartmel B, Molinaro AM, Gordon PB, Leffell DJ, Bale AE, Mayne ST. Host phenotype characteristics and MC1R in relation to early-onset basal cell carcinoma. J Invest Dermatol 2012;132:1272-9.

4. Kwon NH, Kim SY, Kim GM. A case of metastasis squamous cell carcinoma arising from actinic cheilitis. Ann Dermatol 2011;23:101-3.

5. Vieira RA, Minicucci EM, Marques ME, Marques SA. Actinic cheilitis and squamous cell carcinoma of the lip: clinical, histopathological and immunogenetic aspects. An Bras Dermatol 2012;87:105-14.

6. Wood NH, Khammissa R, Meyerov R, Lemmer J, Feller L. Actinic cheilitis: a case report and a review of the literature. Eur J Dent 2011;5:101-6. 